Acta Botánica Mexicana (1994), 28:29-39

\title{
WIGANDIA URENS (HYDROPHYLLACEAE): UN MOSAICO DE RECURSOS PARA SUS INSECTOS HERBIVOROS
}

\author{
Zenon Cano-Santana y Ken Oyama \\ Centro de Ecología, UNAM \\ Apartado Postal 70-275 \\ 04510 México, D.F.
}

\begin{abstract}
RESUMEN
Wigandia urens (Ruiz \& Pavón) H.B.K. (Hydrophyllaceae) es un arbusto común en la Reserva del Pedregal de San Angel, D.F. Esta especie presenta una gran variación morfológica y química en hojas y tallos. Con base en la pubescencia, se pueden reconocer dos tipos de láminas foliares en esta planta: híspidas con tricomas urticantes y glandulares, y lisas con sólo tricomas glandulares. La presencia y densidad de estos tricomas cambian de acuerdo con la edad de las hojas y la heterogeneidad ambiental. Debido a la gran variación que presenta $W$. urens, se puede considerar que los individuos de esta especie representan un mosaico de recursos para los herbívoros que coexisten con ella. En tal contexto se estudiaron las preferencias alimentarias y de asentamiento de los fitófagos más comunes que viven sobre $W$. urens en la Reserva del Pedregal de San Angel. Esto se llevó a cabo con observaciones periódicas en el campo y con experimentos de aceptabilidad. Se encontraron 14 especies de insectos herbívoros asociados a $W$. urens pertenecientes a cinco órdenes (Diptera, Hemiptera, Homoptera, Lepidoptera y Orthoptera). Estos animales presentaron diferentes grados de predilección y de asentamiento sobre $W$. urens.

Los experimentos para conocer las preferencias alimenticias de los herbívoros consistieron básicamente en ofrecer hojas (jóvenes / maduras; híspidas / lisas) de W. urens a diversos herbívoros de manera individual. Las especies utilizadas fueron larvas de dos lepidópteros: Sphinx lugens y Lophoceramica pyrrha, y adultos y ninfas de los ortópteros Sphenarium purpurascens e Ichthyotettix mexicanus. I mexicanus presentó una preferencia por hojas híspidas y maduras de $W$. urens, mientras que $S$. lugens prefirió hojas con ambos tipos de tricomas. Ninfas de $S$. purpurascens y larvas de $L$. pyrrha presentaron una ligera preferencia por hojas jóvenes de $W$. urens. Estos resultados se discuten enfatizando la relevancia de estudiar las interacciones entre herbívoros y plantas considerando a estas últimas como mosaicos de recursos que varían tanto espacial como temporalmente.
\end{abstract}

\section{ABSTRACT}

Wigandia urens (Ruiz \& Pavón) H.B.K. (Hydrophyllaceae) is a common shrub at the Pedregal de San Angel reserve in Mexico City. This species has a great morphological and chemical variation on leaves and stems. It is possible to recognize two types of leaves on plants of $W$. urens: smooth leaves with only glandular trichomes and bristly leaves with urticant hairs and glandular trichomes. The presence and density of trichomes vary according to leaf age and environmental heterogeneity. The great variation of plants of $W$. urens represents a mosaic of resources to herbivores associated to them. In this study, feeding preferences and patterns of establishment of the most common herbivores on plants of $W$. urens in the Pedregal de San Angel were studied. Field observations and acceptability experiments were performed. In this study, we found 14 species of herbivore insects belonging to five different orders (Diptera, Hemiptera, Homoptera, Lepidoptera and Orthoptera). In general, all the species showed different degrees of specificity and preference for different parts of $W$. urens in the field. 
The experiments consisted basically in offering leaves (young / mature; bristly / smooth) of $W$. urens to a diverse number of herbivores in an individual way. Larvae of two lepidoptera, Sphinx lugens and Lophoceramica pyrrha, and adults and nymphs of two orthoptera, Ichthyotettix mexicanus and Sphenarium purpurascens were used in the experiments. I. mexicanus showed a preference for bristly mature leaves of $W$. urens, while $S$. lugens fed both smoot and bristly leaves. Nymphs of $S$. purpurascens and larvae of $L$. pyrrha had a preference for young leaves. These results are discussed emphasizing the relevance of considering plants as mosaics of resources that vary spatially and temporally in studies of plant-herbivore interactions.

\section{INTRODUCCION}

Las plantas pueden ser vistas como mosaicos de recursos que varían temporal y espacialmente en su disponibilidad como alimento para sus herbívoros (McNeill y Southwood, 1978; Whitham, 1981; Schultz, 1983; Karban 1992). Las diferencias morfológicas, químicas y nutricionales encontradas en los individuos de una población de plantas determinan diferentes niveles de interacción con sus predadores a través de tres posibles mecanismos. En primer lugar, la variación hace que algunas plantas sean menos aparentes a sus enemigos naturales incrementando la probabilidad de que éstos lleven a cabo asentamientos 0 decisiones alimenticias inapropiadas. En segundo lugar, las interacciones competitivas entre fitófagos por mejores recursos alimenticios pueden incrementarse influyendo en la sobrevivencia, desarrollo o reproducción de los mismos. Y en tercer lugar, los herbívoros pueden agruparse en sitios específicos de la planta siendo más aparentes y vulnerables a sus propios depredadores (Whitham, 1981; Schultz, 1983; Karban, 1992).

El papel de la variabilidad es bien conocido entre los agrónomos, quienes se han dado cuenta que al reducirla entre las plantas, se elevan algunos herbívoros al nivel de plagas, de modo que actualmente, en los cultivos económicamente importantes se ha incrementado la variación mezclando diferentes variedades de vegetales (Denno y McClure, 1983; Whitham, 1981).

Wigandia urens es un arbusto que se caracteriza por presentar diferencias individuales en la pubescencia de sus hojas, la que se relaciona con la calidad nutricional de las mismas (Cano-Santana, 1987; Cano-Santana y Oyama, 1992a). Este arbusto, dentro de la reserva del "Pedregal de San Angel", es utilizado por 17 especies de herbívoros, todos ellos insectos, la mayoría de los cuales se presentan en la temporada de lluvias, que ocurre de junio a octubre (Carbajal-Moreno, 1975). Las diferencias morfológicas y químicas que se observan en las hojas de $W$. urens, hacen que la especie sea un buen sistema para analizar el papel de la variación foliar en el comportamiento de forrajeo de fitófagos con diferentes estrategias de uso de recursos.

El objetivo de este trabajo es conocer el grado de variabilidad de las preferencias alimenticias y de asentamiento de algunas de las especies de herbívoros más importantes de W. urens.

Descripción de la planta

Wigandia urens (Ruiz \& Pavón) H.B.K. (Hydrophyllaceae) es un arbusto fisonómicamente importante en la reserva. Se caracteriza por presentar en sus hojas dos tipos de tricomas: glandulares y urticantes. Los glandulares son pequeños $(0.7-1.0 \mathrm{~mm}$ de 
largo), con un pedículo compuesto por cuatro o cinco células y una cabeza multicelular que produce una secreción viscosa. Los urticantes son largos $(3.0-6.0 \mathrm{~mm})$, duros y puntiagudos que al ser tocados liberan un líquido que produce dolor y escozor. Los primeros están presentes de manera uniforme en todas las hojas de $W$. urens, en tanto que la densidad de los segundos varía de una hoja a otra, de modo que coexisten dentro de una población, y aun dentro de una sola planta, tanto hojas híspidas (con ambos tipos de tricomas) como lisas (sólo con los glandulares) (Cano-Santana, 1987).

Análisis químicos comparativos en ambos tipos de hojas revelaron que las híspidas presentan una concentración significativamente mayor de nitrógeno, fósforo y agua que las lisas, aunque en ambos tipos se registraron los mismos compuestos secundarios (flavonoides, fenoles, esteroides y glucósidos) (Cano-Santana, 1987; Cano-Santana y Oyama, 1992a). Asimismo, se encontró que la presencia y densidad de tricomas urticantes en las hojas está relacionada con condiciones de baja disponibilidad de agua en el ambiente (Cano-Santana, 1987; Cano-Santana y Oyama, 1992a).

\section{Herbívoros de Wigandia urens}

Carbajal-Moreno (1975) registró 17 especies de insectos herbívoros asociados a $W$. urens. Sin embargo, en nuestro estudio únicamente encontramos las siguientes 14 especies: los ortópteros Sphenarium purpurascens Charpentier (Acrididae), Ichthyotettix mexicanus Saussure (Acrididae) y Oecanthus sp.; un hemíptero del género Collaria (Miridae); los homópteros Aconophora pallescens Stal. (Membracidae), Myzus persicae (Sulzer) (Aphididae), Graphocephala sp. y una especie no identificada; los lepidópteros Sphinx lugens Walk. (Sphingidae), Autographa biloba (Steph.) (Noctuidae, Phytometrinae), Baratra configurata Walk., Lophoceramica pyrrha (Druce) (Noctuidae, Noctuinae) y Sabulodes matrona Druce (Geometridae); y un díptero minador del género Liriomyza (Agromyzidae). De éstos, sólo Collaria sp. y M. persicae se presentan todo el año, ya que la mayoría se concentra en la estación de lluvias. Los más abundantes son S. purpurascens, L. pyrrha, Collaria sp. y M. persicae, y los más especializados en utilizar a $W$. urens son S. lugens, A. pallescens e I. mexicanus (Cano-Santana, 1987).

\section{METODOLOGIA}

Este trabajo se llevó a cabo en la reserva "El Pedregal de San Angel" ubicada en el campus de la Universidad Nacional Autónoma de México en la ciudad de México. Esta reserva de 124.5 ha está cubierta por una vegetación de matorral xerófilo (Rzedowski, 1954). La temperatura media anual es de $15.5^{\circ} \mathrm{C}$ y la precipitación promedio anual es de $870 \mathrm{~mm}$ (Valiente-Banuet y Luna-García, 1990). Existe una marcada estacionalidad, presentándose una temporada seca de noviembre a mayo y una lluviosa de junio a octubre.

Observaciones de campo

Se realizaron visitas periódicas al Pedregal de San Angel para conocer la presencia de insectos herbívoros en $W$. urens. Se registró el tipo de hojas en las que se encontraron de acuerdo con la edad (yema foliar, hojas jóvenes, intermedias y maduras), pubescencia 
(híspidas o lisas), así como con el tamaño de la planta. La edad de las hojas se estimó por su posición en las ramas, su tamaño y la coloración de las cabezuelas de los tricomas glandulares. Adicionalmente, se hicieron observaciones sobre las posibles especies alternativas que $S$. purpurascens, $L$. pyrrha y $S$. lugens usan como alimento para determinar el grado de especificidad de estos insectos con respecto a $W$. urens.

Preferencias alimenticias

Para conocer el efecto inmediato de la edad y de la pubescencia de las hojas de $W$. urens sobre la selectividad o preferencia que tienen sus herbívoros, se realizaron varios experimentos utilizando larvas de L. pyrrha y S. lugens, y ninfas y adultos de ambos sexos de S. purpurascens colectados en el Pedregal de San Angel, así como adultos de ambos sexos de I. mexicanus colectados en el Derrame del Chichinautzin (km 72 de la autopista México-Cuernavaca), debido a su escasez en la reserva del Pedregal. Estas especies fueron elegidas considerando (i) su abundancia en el Pedregal o localidades alternativas, (ii) su tamaño, (iii) la relativa facilidad en su manipulación experimental, y (iv) su comportamiento de forrajeo (e.g., masticadores).

Los insectos previamente sometidos a ayuno durante 24 horas, fueron colocados en cajas de plástico de $8 \mathrm{~cm}$ de altura por $15 \mathrm{~cm}$ de diámetro con un trozo de algodón humedecido para evitar su desecación. Las hojas utilizadas se colectaron frescas en el campo y se cortaron en pedazos de $36 \mathrm{~cm}^{2}(6 \times 6 \mathrm{~cm})$ para el experimento. Para cada tratamiento se hicieron de 6 a 7 réplicas de acuerdo con la disponibilidad de los animales en el campo y con las condiciones de los mismos después del ayuno. Los diseños experimentales fueron los siguientes:

Diseño 1. Un trozo de hoja de un solo tipo se usó en cada dispositivo de modo que el herbívoro tuviese una sola opción para alimentarse.

Diseño 2. Dos trozos de hojas, uno de cada tipo se colocaron simétricamente en cada dispositivo de modo que el insecto tuviese dos opciones para alimentarse.

Diseño 3. Cuatro trozos de hojas se colocaron por dispositivo probando simultáneamente el efecto de la edad y la pubescencia foliares. Las larvas fueron alimentadas con hojas jóvenes híspidas, jóvenes lisas, maduras híspidas y maduras lisas.

Estos experimentos se delinearon tratando de cubrir las diferentes posibilidades alimenticias de los animales. En el diseño 1, en donde el insecto sólo tiene una opción de comida, el objetivo es conocer si puede o no utilizar determinado follaje. En los diseños 2 y 3 , se trata de conocer la preferencia del herbívoro cuando tiene dos y cuatro disyuntivas bajo la suposición de que éste tiene la capacidad de escoger su alimento más apetecido. Los experimentos se realizaron durante diferentes intervalos de tiempo (1 a 24 horas) para determinar las preferencias inmediatas de los fitófagos. Estas observaciones se realizaron bajo la suposición de que un herbívoro tenderá a consumir en seguida un tipo de comida si se encuentra dentro de su prioridad alimenticia. No se realizó ningún registro después de 24 horas, ya que pasado este tiempo, los trozos de hoja empezaron a secarse.

En los experimentos para definir la preferencia de hojas de diferente edad se utilizaron hojas jóvenes y maduras con la misma densidad de tricomas, en tanto que en los que se estimaba el efecto de la pubescencia se emplearon hojas híspidas y lisas de la misma edad. 
Para calcular la cantidad de hoja consumida se dibujó el contorno de la misma y se midió su superficie en un medidor de áreas foliares. La relación entre esta medida y la superficie del trozo de hoja original se tomó como el porcentaje de área comida. Sólo para el experimento con $S$. lugens se empleó una balanza para obtener el peso fresco de hojas usadas como alimento. Para ello, se determinó el porcentaje de desecación natural empleando trozos de hojas sin larvas como control; el peso de material consumido se obtuvo utilizando el método gravimétrico (Waldbauer, 1968).

Todos los experimentos se llevaron a cabo dentro de un invernadero bajo las siguientes condiciones: temperatura de $20^{\circ} \mathrm{C} \pm 5^{\circ} \mathrm{C}$, humedad relativa promedio de $70 \%$ y un fotoperíodo natural.

Las preferencias de asentamiento sólo pudieron ser calculadas para Collaria sp. y para $M$. persicae, tomando como base para el áfido únicamente los datos del mes de noviembre cuando éste fue más abundante. La cuantía de estos insectos se comparó en tablas de contingencia clasificadas de acuerdo con la edad y la pubescencia de la planta. Los valores observados y calculados se contrastaron con pruebas de chi-cuadrada (Zar, 1974). Los datos obtenidos a partir de los experimentos con el diseño 1 fueron sometidos a una prueba de $t$ para muestras independientes comparando tipos de hoja como factores (hojas lisas o híspidas y hojas jóvenes o maduras). Los datos obtenidos con el diseño 2 se analizaron con una prueba de $t$ para muestras pareadas comparando tipos de hojas pero controlando el sujeto experimental, ya que ambas muestras foliares se colocaron en el mismo dispositivo experimental. Los datos obtenidos en el diseño 3 se compararon con un análisis de varianza de una vía utilizando el tipo de hojas (cuatro niveles) como factor (Zar, 1974). Cada tratamiento se realizó de manera independiente y los dispositivos (cajas de plástico) usados fueron colocados en una mesa común. El orden y la posición de cada caja fue elegida aleatoriamente. Los herbívoros también se seleccionaron al azar. Ningún individuo se utilizó dos veces en el mismo experimento o en experimentos diferentes. La posición de cada trozo de hoja en los diseños 2 y 3 se determinó aleatoriamente dentro de cada dispositivo.

\section{RESULTADOS}

Observaciones de campo

Las observaciones más importantes hechas en las visitas continuas al campo durante un año fueron las siguientes. Sphinx lugens no se encuentra en yemas foliares u hojas muy jóvenes ni en plantas de menos de $0.5 \mathrm{~m}$ de altura. Autographa biloba y Baratra configurata coexisten a veces en individuos pequeños sobre yemas foliares y hojas jóvenes. Por otro lado, mientras $L$. pyrrha y Collaria sp. nunca se observaron en los de talla pequeña, menores de $0.5 \mathrm{~m}, M$. persicae se halló en hospederos de todos tamaños. Larvas de $L$. pyrrha se encontraron principalmente en hojas maduras con ambos tipos de tricomas. Collaria sp. fue más común en yemas foliares, hojas jóvenes e intermedias pero sin pelos urticantes. En los pocos registros de $I$. mexicanus, éste utilizaba plantas de todos tamaños siendo más común en hojas híspidas. A $S$. purpurascens también se le encontró en individuos de tallas variadas agregándose sobre las hojas maduras (Fig. 1). 

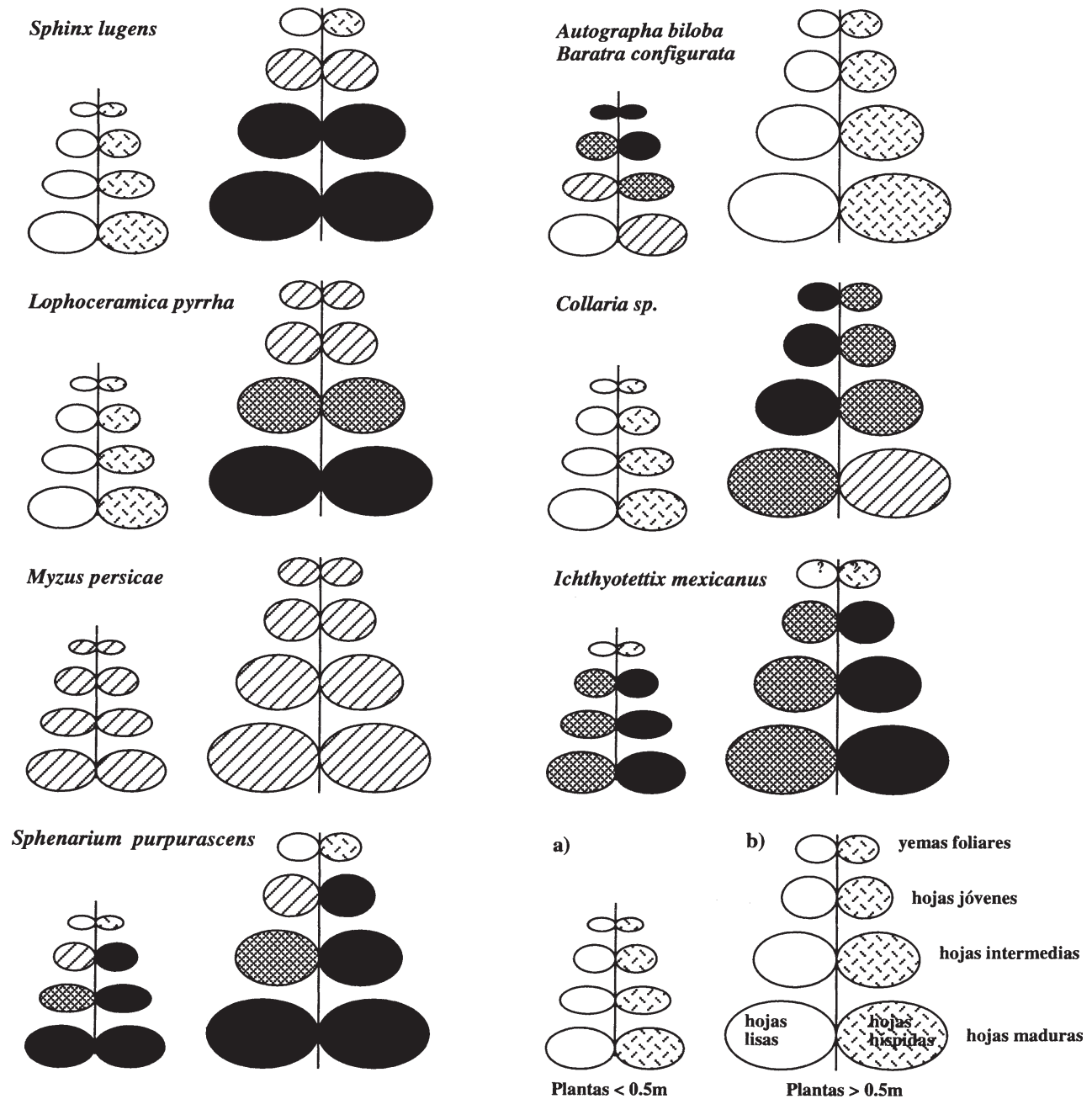

Fig. 1. Representación diagramática de las preferencias alimenticias y de asentamiento de ocho especies de herbívoros por hojas híspidas y lisas de diferente edad (yemas, jóvenes, intermedias y maduras) de Wigandia urens en el Pedregal de San Angel. Se muestran plantas de dos tamaños a) $<0.5 \mathrm{~m} \mathrm{y} \mathrm{b)}>0.5 \mathrm{~m}$. La intensidad de preferencia está representada como: alta (sombreado), intermedia (cuadriculado), baja (líneas) y nula (en blanco). Esta figura resume la información acumulada en observaciones de campo (cuadro 1 y observaciones no mencionadas en este trabajo), los resultados experimentales previamente descritos en este estudio, e información publicada con anterioridad (CanoSantana, 1987; Cano-Santana y Oyama, 1992a). 
La mayor diversidad y abundancia de herbívoros ocurrió de junio a septiembre (Cuadro 1). Durante los meses de octubre y noviembre los más numerosos fueron Collaria sp. (38\% de las hojas), S. purpurascens (7\%), M. persicae $(2 \%)$ y L. pyrrha (1\%). Al comparar el borde y el interior de la reserva, se encontró una mayor abundancia y diversidad de estos insectos en los bordes. Las preferencias de asentamiento sólo pudieron calcularse para Collaria sp. y para $M$. persicae. Collaria sp. mostró propensión por hojas lisas de $W$. urens, tanto jóvenes como intermedias $\left(\mathrm{x}^{2}=73.72 ; \mathrm{P}<0.001\right)$. Por su parte, el áfido $M$. persicae no presenta predilección por ningún tipo particular de hojas de diferente edad $\left(\mathrm{X}^{2}=4.72 ; \mathrm{P}<0.05\right)$ ni con diferente pubescencia $\left(\mathrm{X}^{2}=1.73 ; \mathrm{P}<0.05\right)$.

Cuadro 1. Abundancia y riqueza de especies de herbívoros encontrados sobre Wigandia urens en dos temporadas (1 de octubre y 14 de noviembre) y dos zonas (interior y borde) del Pedregal de San Angel, D.F. En todos los casos se muestrearon 288 hojas.

\begin{tabular}{|c|c|c|c|c|}
\hline \multirow[b]{3}{*}{ Especies $^{1}$} & \multicolumn{4}{|c|}{ Número de registros } \\
\hline & \multicolumn{2}{|c|}{1 octubre } & \multicolumn{2}{|c|}{14 de noviembre } \\
\hline & interior & borde & interior & borde \\
\hline Collaria sp. & 83 & 127 & 142 & 90 \\
\hline Sphenarium purpurascens & 5 & 44 & 8 & 25 \\
\hline Myzus persicae & 4 & 1 & 7 & 19 \\
\hline Aconophora pallescens & --- & 1 & --- & 1 \\
\hline Lophoceramica pyrrha & 4 & 10 & --- & 1 \\
\hline Sphinx lugens & --- & 1 & --- & --- \\
\hline Baratra configurata & 1 & --- & --- & --- \\
\hline Total & 97 & 184 & 157 & 136 \\
\hline
\end{tabular}

${ }^{1}$ Otras especies registradas en hojas de Wigandia urens en otras épocas del año pero no observadas en este muestreo particular fueron: Graphocephala sp. y una especie no identificada (Homoptera), Autographa biloba (Lepidoptera), Sabulodes matrona (Lepidoptera), Liriomyza sp. (Diptera), Oecanthus $\mathrm{sp}$. e Ichthyotettix mexicanus (Orthoptera).

Preferencias alimenticias

Los experimentos encaminados a tal objetivo señalan los siguientes patrones de selectividad (Cuadro 2). Machos y hembras de I. mexicanus no presentan preferencias entre hojas jóvenes y maduras de $W$. urens. Sin embargo, a nivel de pubescencia foliar, los machos comieron mayor cantidad de hojas híspidas a las $6 \mathrm{~h}$ de tratamiento, hecho que no ocurrió con las hembras. El porcentaje de área foliar consumida (\% AF) por los machos de esta especie no difirió significativamente a las 12, 18 y 24 horas. A las 6 horas, los machos habían dispuesto de $2.3 \pm 0.4 \%$ AF en hojas híspidas y $0.3 \pm 0.3 \%$ AF en hojas lisas; a las 24 horas el uso de las primeras se mantuvo igual, mientras que el de las segundas se incrementó a $1.0 \pm 0.8 \%$ AF. Los machos de $I$. mexicanus prefieren hojas 
Cuadro 2. Preferencias alimenticias de cuatro especies de herbívoros hacia hojas de diferente edad y pubescencia de Wigandia urens. El número de réplicas en todos los diseños fue de 6 organismos con excepción del experimento con Sphinx lugens en el que se utilizaron 7 individuos.

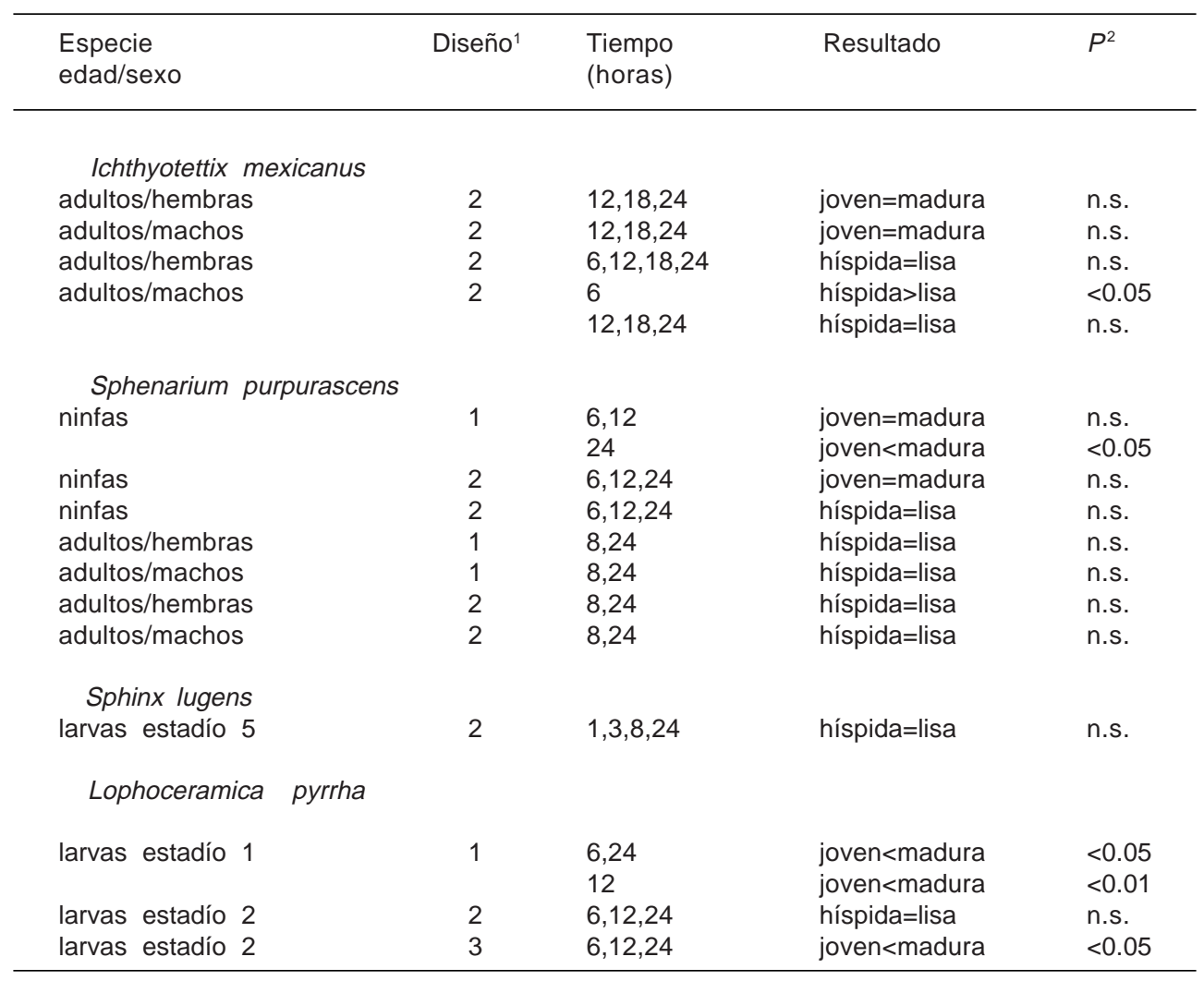

1Ver métodos

${ }^{2}$ Prueba de $t$ para muestras independientes (diseño 1); prueba de $t$ para muestras pareadas (diseño 2); análisis de varianza de una vía (diseño 3); n.s.= no significativo a $P<0.05$.

híspidas en lugar de lisas (diseño 2). Las hembras de esta especie utilizan mayor cantidad de alimento que los machos. A las 24 horas éstas consumieron $13.6 \pm 4.2$ y $9.5 \pm 2.7 \%$ AF de hojas híspidas y lisas respectivamente, lo cual puede estar relacionado con las diferencias de tamaño entre los sexos, pues las hembras miden de 2.8 a $3.2 \mathrm{~cm}$ de largo mientras que los machos 1.7 a $2.0 \mathrm{~cm}$ (Cano-Santana, 1987).

Las ninfas y los adultos de $S$. purpurascens comieron por igual hojas híspidas y lisas. Con base en los experimentos de diseño 2 se encontró que no hay preferencias de parte de las ninfas del tercer estadio de esta especie por una edad particular de hoja. Sin embargo, en el diseño 1, las ninfas del primer estadio consumieron más hojas maduras 
a las 24 horas de tratamiento (hojas maduras $=7.35 \pm 0.76$; hojas jóvenes $=3.28 \pm 0.66 \%$ AF consumida). Las larvas del quinto estadio de $S$. lugens, por su parte, no presentaron predilección alguna al ofrecerles hojas con diferente pubescencia, en tanto que las larvas de L. pyrrha se alimentaron de hojas maduras independientemente de la pubescencia. Al utilizar el diseño 3 para comparar los cuatro tipo de hojas, se encontró nuevamente un mayor consumo de hojas maduras.

En la figura 1 se sintetizan las preferencias alimenticias y de asentamiento de los ocho herbívoros más comunes: S. lugens, L. pyrrha, Ichthyotettix mexicanus, $S$. purpurascens, A. biloba, B. configurata, Collaria sp. y M. persicae con base en los resultados de los ensayos experimentales, los muestreos de herbívoros y las observaciones de campo. La información contenida en la figura fue complementada con datos previamente publicados y otros no mencionados en este trabajo (Cano-Santana, 1987; Cano-Santana y Oyama, 1992a; Z. Cano-Santana, obs. pers). El diagrama ilustra la forma en que el mosaico de recursos presente en individuos de $W$. urens determina la ocupación y utilización de diferentes partes por los herbívoros asociados a esta planta.

\section{DISCUSION}

Wigandia urens es una planta muy variable en sus características físicas y químicas (Ochoa, 1985; Cano-Santana y Oyama, 1992a). Sus hojas no sólo presentan variación por efecto de la edad, sino también por la densidad de tricomas urticantes. Al momento, sólo se sabe de la mayor riqueza nutricional de las hojas híspidas respecto a las lisas (CanoSantana, 1987; Cano-Santana y Oyama, 1992a). En términos generales, se desprende que cada especie de herbívoro tiene distintas predilecciones hacia plantas de edades y tipos diferentes de hojas. Por ejemplo, mientras $A$. biloba y $B$. configurata se asientan con más frecuencia en individuos jóvenes, S. lugens, L. pyrrha y Collaria sp. nunca se encuentran en los de esa edad. Asimismo, Collaria sp. prefiere hojas jóvenes sobre las maduras, y a las híspidas sobre las lisas, mientras que $S$. purpurascens consume principalmente hojas maduras y probablemente las híspidas. Por otra parte, los machos del chapulín I. mexicanus, anteponen como alimento las hojas híspidas sin importarle su edad y $S$. lugens da prioridad a las maduras independientemente de la pubescencia foliar. Un caso interesante es el de M. persicae que no presenta preferencias marcadas hacia hojas o plantas de determinada edad (ver Fig. 1). Cabe mencionar que estos resultados han sido confirmados con observaciones continuas de campo realizadas durante varios años (1987-1992) (Z. CanoSantana, datos no publicados).

Una de las conclusiones más interesantes de estos resultados es que todos los herbívoros de $W$. urens, con excepción de Collaria sp., no tienen problemas para enfrentarse con los tricomas urticantes foliares. Cultivos de larvas de S. lugens y L. pyrrha realizados con dietas de hojas híspidas y lisas han demostrado que, en general, la tasa de crecimiento es más alta y la duración del estado larvario se acorta bajo una dieta de hojas híspidas (Cano-Santana, 1987; Cano-Santana y Oyama, 1992b, en prensa). Esto haría suponer que los insectos que se alimentan de hojas híspidas tienen muchas ventajas, dada la mayor riqueza nutricional de las mismas. Sin embargo, el hecho de que la proporción existente entre hojas lisas e híspidas en la población de $W$. urens de la reserva del Pedregal no se mantiene constante, determina que las relaciones planta-insecto se mantengan sincronizadas temporalmente con la disponibilidad de cierto tipo de hojas y la presencia de los herbívoros (Cano-Santana, 1987; Cano-Santana y Oyama, 1992a). 
Wigandia urens en el Pedregal se manifiesta como un mosaico de posibilidades de alimentación, tanto espacial como temporalmente, que influye de diferentes maneras sobre sus herbívoros. Las preferencias alimenticias de éstos son un indicador de la posible variación en compuestos químicos tóxicos o en la calidad nutricional que afecta las hojas.

Esta diversidad de estrategias de preferencias alimentarias demuestra cómo cada especie de herbívoro se asocia de manera diferente a un mosaico de recursos. Whitham (1983) señala que en el sistema Populus angustifolia y áfidos de las agallas del género Pemphigus, el patrón de asentamiento de los herbívoros colonizadores refleja un mosaico de resistencia del hospedero que necesariamente afecta la adecuación de los áfidos. En el caso de $W$. urens el mosaico cobra más sentido, puesto que dificulta la localización del alimento simultáneamente a todos sus herbívoros, ya que ninguno de ellos puede explotar con la misma eficiencia todas las partes del mosaico. Por otro lado, la energía gastada por el herbívoro para la búsqueda del alimento se incrementa conforme la heterogeneidad de éste último es mayor. Cuando la disponibilidad de las partes comestibles disminuye, los individuos o las especies que utilizan las mismas partes del mosaico compiten entre sí sobre todo en condiciones de alto tamaño poblacional.

La agrupación de herbívoros en partes específicas del mosaico (como ocurre con las colonias de L. pyrrha) provoca que éstos puedan ser más aparentes para depredadores y parasitoides afectando las densidades de sus poblaciones y de sus futuras expectativas de vida (Attsat y O'Dowd, 1975; Feeny, 1976; Whitham, 1980). La existencia de un mosaico de recursos vegetales puede influir en el grado de especialización de los insectos hacia un tipo de hoja, incrementar el tiempo de forrajeo y la compatibilidad fenológica con la planta huésped (Schulz et al., 1982; Karban, 1992).

La variabilidad química foliar está estrechamente relacionada con el grado de desarrollo y de la pubescencia particular de cada hoja y con la conformación de un mosaico único representado por cada planta individual. Esta heterogeneidad de la población obstaculiza el ataque de los herbívoros con diferente grado de especialización. Sin embargo, el verdadero valor de esta variabilidad para el organismo vegetal sólo puede ser puesto en evidencia con experimentos en el campo, en los que se pueda establecer la relación entre el grado de variabilidad foliar y los niveles de daño producidos a cada individuo.

\section{AGRADECIMIENTOS}

Los autores de este trabajo agradecen a Sonia Careaga y Ricardo Vergara por su ayuda en el trabajo de laboratorio y de campo; a Ana Mendoza por facilitar las instalaciones donde se llevaron a cabo los experimentos y a Rosa María Murillo por su cuidadosa y constructiva revisión del manuscrito original.

\section{LITERATURA CITADA}

Atsatt, P. R. y D. J. O'Dowd. 1976. Plant defense guilds. Science 193: 24-29.

Cano-Santana, Z. 1987. Ecología de la relación entre Wigandia urens (Hydrophyllaceae) y sus herbívoros en el Pedregal de San Angel, D.F. (México). Tesis Profesional. Facultad de Ciencias, Universidad Nacional Autónoma de México. México, D.F. 157 pp. 
Cano-Santana, Z. y K. Oyama. 1992a. Variation in leaf pubescence of Wigandia urens (Hydrophyllaceae) and its implications for herbivory. Oecologia 92: 405-409.

Cano-Santana, Z. y K. Oyama. 1992b. Tricomas foliares, calidad del alimento y eficiencias de alimentación y crecimiento de Lophoceramica pyrrha (Lepidoptera, Noctuidae). Southwest. Entomol. 17: 333-339.

Cano-Santana, Z. y K. Oyama. (en prensa). Crecimiento de Sphinx lugens (Lepidoptera: Sphingidae) bajo una dieta de hojas híspidas y lisas de Wigandia urens. Folia Entomol. Mex. 87.

Carbajal-Moreno, L. 1975. Estudio ecológico de los insectos que viven en Wigandia caracasana HBK de una zona del Pedregal de San Angel, D.F. Tesis Profesional. Facultad de Ciencias, Universidad Nacional Autónoma de México. México, D.F. 103 pp.

Denno, R. F. y M. S. McClure (eds.). 1983. Variable plants and herbivores in natural and managed systems. Academic Press. Nueva York. 717 pp.

Feeny, P. 1976. Plant apparency and chemical defense. Recent Adv. Phytochem. 10: 1-40.

Karban, R. 1992. Plant variation: its effects on populations of herbivorous insects. In: Fritz, R. S. y E. L. Simms (eds.). Plant resistance to herbivores and pathogens. Ecology, evolution and genetics. The University of Chicago Press. Chicago. pp. 195-215.

McNeill, S. y T. R. E. Southwood. 1978. The role of nitrogen in the development of insect plant relationships. In: Harborne, J. B. (ed.). Biochemical aspects of plant and animal coevolution. Academic Press. Nueva York. pp. 77-98.

Ochoa G., S. 1985. Hydrophyllaceae. In: Rzedowski, J. y G. C. Rzedowski (eds.). Flora fanerogámica del Valle de México. Vol. II. Instituto de Ecología. México. pp. 262-267.

Rzedowski, J. 1954. Vegetación del Pedregal de San Angel (D.F., México). An. Esc. Nac. Cien. Biol. Méx. 8: 59-129.

Schultz, J. C. 1983. Habitat selection and foraging tactics of caterpillars in heterogeneous trees. In: Denno, R. F. y M. S. McClure (eds.). Variable plants and herbivores in natural and managed systems. Academic Press. Nueva York. pp. 61-90.

Schultz, J. C., P. J. Nothnangle y I. T. Baldwin. 1982. Seasonal and individual variation in leaf quality of two northern hardwoods tree species. Am. J. Bot. 69: 753-759.

Valiente-Banuet, A. y E. de Luna. 1990. Una lista florística actualizada para la reserva del Pedregal de San Angel, México D.F. Acta. Bot. Mex. 9:13-30.

Waldbauer, G. P. 1968. The consumption and utilization of food by insects. Adv. Insect Physiol. 5: 229-288.

Whitham, T. G. 1980. The theory of habitat selection: examined and extended using Pemphigus aphids. Am. Nat. 115: 449-466.

Whitham, T. G. 1981. Individual trees as heterogeneous environments. Adaptation to herbivory or epigenetic noise? In: Denno, R. F. y H. Dingle (eds.). Insect life history patterns: habitat and geographic variation. Springer-Verlag. Nueva York. pp. 9-27.

Whitham, T. G. 1983. Host manipulation of parasites: within-plant variation as a defense against rapidly evolving pests. In: Denno, R. F. y M. S. McClure (eds.). Variable plants and herbivores in natural and managed systems. Academic Press. Nueva York. pp. 15-41.

Zar, J. H. 1974. Biostatistical analysis. Prentice-Hall. Englewood Cliffs, Nueva Jersey. 620 pp. 